\title{
Coupling Coordination between Technology Transfer in Universities and High-Tech Industries Development in China
}

\author{
Haining Fang $\mathbb{D}$, ${ }^{1}$ Qing Yang $\mathbb{D},{ }^{1,2}$ Jinmei Wang $\mathbb{D}{ }^{2}$, and Xingxing Liu $\mathbb{D}^{2}$ \\ ${ }^{1}$ School of Management, Wuhan University of Technology, Wuhan 430070, China \\ ${ }^{2}$ School of Safety Science and Emergency Management, Wuhan University of Technology, Wuhan 430070, China \\ Correspondence should be addressed to Jinmei Wang; wangjinmei@whut.edu.cn
}

Received 9 April 2021; Revised 18 June 2021; Accepted 28 June 2021; Published 7 July 2021

Academic Editor: Miguel Fuentes

Copyright ( 2021 Haining Fang et al. This is an open access article distributed under the Creative Commons Attribution License, which permits unrestricted use, distribution, and reproduction in any medium, provided the original work is properly cited.

Only through successful transformation and application in industries, technology achievements in universities can promote economic and social development. Meanwhile, technological progress in high-tech industries depends in part on universities' technology achievements. Coupling coordination between technology transfer in universities (TTU) and high-tech industries development (HTID) is of great significance to the sustainable development of the regional sci-tech innovation system. In this paper, the coupling mechanism of TTU and HTID is analyzed and comprehensive evaluation index systems are established by using the evaluation indicator to screen and assign weights based on information contribution rate. The coupling coordination index (CCI) and productivity index (PI) are introduced to derive the spatiotemporal characteristics of the coupling and coordination of TTU and HTID from 2010 to 2019 in China and analyze its influence factors by System-Generalized Method of Moments (SYS-GMM). The results show the following: (1) the overall national TTU-HTID CCI shows a gradual upward trend and large regional differences; (2) CCI of most provinces is increasing, but the differences are widening; (3) the national TTU PI is in a state of volatility and decline, but the ability of TTU is still much higher than the level of HTID; (4) technological innovation in high-tech industries, high-tech industry technology absorption capacity, high-tech industry development level, industry-university cooperation, and technological finance development have significant roles in promoting the coupling coordination of TTU-HTID. Meanwhile, the sci-tech innovation level in universities, technology transferability in universities, government support, development of sci-tech intermediary, and development of other research institutions have no significant impact.

\section{Introduction}

Both universities and industries are the key actors of the innovation system of any country, contributing to knowledge production and transformation [1]. Collaborations between companies and universities are critical drivers of the innovation economy [2]. Guiding the integration of the industrial chain and innovation chain is the key to promoting industries to take a leading position in the global value chain and form a core and sustainable competitiveness. Compared with low- and medium-tech industries, high-tech industries (HTI) are mainly characterized by knowledge and technology intensity and marked by technological innovation [3]. Some studies show that $40 \%$ of the companies were attached to universities or research institutions [4].
Meanwhile, universities are the birthplace of advanced scientific knowledge and the important incubators of cutting-edge sci-tech [5].

According to statistics from "China Universities' Science and Technology Statistical Data Collection 2019" (statistics for 2018) by Ministry of Education of the People's Republic of China and "China Intellectual Property Statistical Yearbook 2018" by China National Intellectual Property Administration, the number of patents applied by Chinese universities in 2018 reached 310,276 and increased 72.5\% year-on-year, and invention patents accounted for $61.5 \%$ of the three types of patents, which was much higher than the domestic average of $14.8 \%$. However, statistics from "China Intellectual Property Survey Report 2018" by China National Intellectual Property Administration show that the effective 
patent implementation rate of Chinese universities in 2018 is $15.1 \%$, far below the national average of $52.6 \%$. Furthermore, the effective patent industrialization rate of universities is only $3.6 \%$, which is much lower than the national average of $36.3 \%$. Although sci-tech innovation capacity in Chinese universities has been greatly improved, the transfer rate is still low, and the proportion of achievements transformed into industrial development is lower.

Universities play an important role as a source of fundamental knowledge and, occasionally, industrially relevant technology in modern knowledge-based economies [6]. But only through successful transformation and application in industries, technology achievements in universities can promote economic and social development. Thus, university-industry collaboration (UIC) has emerged as a sustainable enabling solution for technology transfer and a booster for new inventions [7, 8].

In order to improve the efficiency of TTU, the United States formulated the Bayh-Dole Act in 1980, which entrusted the ownership of the achievements generated by financial support to universities, encouraged universities and scientists to transfer their achievements independently, and solved the rule predicament [9, 10]. It has not only greatly promoted the number of patent applications in universities of the United States [11] but also transferred patent achievements in large numbers and become an important driving force for the development of HTI and the revitalization of regional economy [12]. The benefits from university-industry collaborations for businesses and universities are reciprocal [13]. It not only reduces the cost of internal R\&D in enterprises [14] but also provides economic support and an industrial platform for the R\&D development of universities $[15,16]$. Stanford and Berkeley's promotion of the development of Silicon Valley is the best example of the coupling development of TTU and HTI [17], which has been imitated by many countries and regions [18]. To solve the key technical problems in the high-tech industries development (HTID) and strengthen the construction of regional technological innovation system, enterprises, universities, governments, and other parties participate in the establishment of a collaborative innovation platform with the triple or quadruple helix structure $[19,20]$. All the platforms accelerate the integration process of the industry chain and innovation chain and strengthen the coupling coordination between technology transfer in university (TTU) and HTID, which can reinforce regional technological innovation driving development, promote the progress of the core and key technology, and advance national innovation system.

However, in China, the contradiction of unbalanced development inadequate performance is more obvious in the field of technology and industry. There is a bigger difference among the different areas in the energy of TTU, the scale of the technology market, and the level of HTID, which led to unbalanced and insufficient coupling coordination between TTU and HTID. This paper studies the level of coupling coordination in different regions and provinces in the past ten years. It also compares the development level of TTU and HTID across the country, which can supplement the level of coupling and coordination. Finally, it also studies the factors that affect the level of coupling and coordination. We not only established the TTU-HTID Coupling System and comprehensive evaluation index systems based on information contribution rate analyzing the spatiotemporal characteristics of the coupling and coordination of TTU and HTID from 2010 to 2019 but also estimated the influencing factors of coupling and coordination by SYS-GMM.

The rest of this article is organized as follows. Section 2 discusses related supporting literature. Section 3 designs the calculation model. Section 4 carries out a numerical analysis. Section 5 summarizes the full text.

\section{Literature Review}

2.1. TTU. Technology transfer, also called technology commercialization [21], is the process of transferring scitech achievements into marketable products and services $[22,23]$. Research on TTU mainly focuses on mechanism model [24], performance evaluation [25], influencing factors [26], value assessment [27], and UIC [28]. Derrick discovered that the flexibility of policies to meet the needs of researchers and open and transparent rewards oriented to the collective are crucial to the transformation of scientific and technological achievements [29]. Blohmke proposed that technology transfer should emphasize the purpose of economic development and the performance of technology, and the evaluation of technology complexity should be incorporated into the technology transfer mechanism [30]. Soares et al. relied on data from a recent survey conducted by the Brazilian Innovation and Technology Transfer Managers National Forum (FORTEC Innovation Survey) and explored how the interplay of the quality of university regulations and regional economic development impacts new patent applications and licensing agreements in the context of Brazilian universities [31].

2.2. HTI. The high-tech industry is one of the most important industries in a knowledge-based economy [32]. Liang proposed that high-tech industries with high investment, high growth, high yield, and high risk should have the following general characteristics. They have (1) a high degree of uncertainty, (2) high value with regard to human resources, and (3) a highly correlated value of intangible assets [33]. According to the explanation from China's National Bureau of Statistics of the People's Republic of China, hightech industries can be defined as a set of enterprises that use high technology as their foundation and execute intense R\&D activities, which include six types of manufactures: medicines; aircraft and spacecraft, and related equipment; electronic equipment and communication equipment; computers and office equipment; medical equipment and measuring instrument; electronic chemicals [34].

Research on high-tech industry focuses on innovation performance [35], agglomeration effect [36], and influencing factors [37]. These researches showed that internationalization [38], technology diversification of industry level [39], strengthening intellectual property rights [40], foreign 
investment [41], the different directions of external knowledge search [42], institutional elements, and market conditions [43] all can contribute to the performance of HTID.

2.3. The Relationship between TTU and HTID. There are also extensive studies about the interactive relationship between university sci-tech innovation and industrial development, which introduces factors that influence the interaction between the two and technology transfer institutions. University management [44], financial and knowledge obstacles [45], organizational proximity [46], and experience accumulated [47] can affect the interactive performance between TTU and HTID. The interaction between TTU and HTID depends not only on their capacity of reciprocal effective adaptation over time but also on the ability to adapt proactively to social and environmental development [48]. To mitigate such cognitive, geographical, organizational, and social distance, a growing number of intermediary organizations, such as Technology Transfer Offices (TTOS), University Incubators (UIs), and Collaborative Research Centers (CRCs), have been established [49]. Brescia et al. studied the organizational structure of technology transfer offices in the world's top 200 universities, emphasizing the role of external, internal, and mixed organizational models [50]. Cassia et al. analyzed 46 entrepreneurship research centers around the world and believed that external stakeholders of knowledge transfer should enrich and support the performance of scientific research [51]. Bikard and Marx called a geographic concentration of patenting by firms in a specialized technical field as hubs. They highlighted the role of hubs of industrial $\mathrm{R} \& \mathrm{D}$ as an interface between academic science and industrial technologies [52]. Goraczkowska Jadwiga found incubators contribute to an increase in the introduction of product innovations by enterprises and in conducting R\&D activities [53].

2.4. The Influences between TTU and HTID. These two systems of TTU and HTID are mainly coupled in the form of technology transfer, technology licensing, technology investment, and university-industry collaboration (UIC). The coupling contents include technology, knowledge, information, products, market, capital, human resources, facility, and other elements. Industry-university cooperation (cooper) is the link between TTU and HTID and represents the scale of their cooperation. For the HTID system, technology innovation is the basis of its survival. Through interactive cooperation with universities, it can take advantage of the rich accumulated knowledge and research human resources to transfer the scitech achievement to industry technology, effectively reduce the risk of industries R\&D input, and save the cost in basic research and applied research [54]. The university sci-tech innovation level (Utech) has a direct impact on the output of scientific and technological achievements in universities, is an important part of the regional scientific and technological innovation system, and promotes HTID.

As for the TTU system, HTID provides human power, facility, capital, and market for the incubation and application of sci-tech achievements [55], which reflects the last link that realizes the value. At the same time, HTID provides a training platform for university researchers to improve their practical abilities. The higher the High-tech industry technology absorption capacity (Habsorb), the stronger the ability of the industry to identify, digest, integrate, and use external sci-tech for technological innovation, and the more it can promote TTU. The higher the Technological innovation level in high-tech industries (Htech), the greater the demand for technological innovation in the industry, and the easier it is to promote the coupling coordination of TTU and HTID.

The coordinated development of the TTU-HTID coupling system requires favorable external conditions, including regional economic development level [56], infrastructure construction [57], human capital accumulation [58], market-opening level [59], etc. Both the system of TTU and HTID not only depend on external conditions but also influence the external conditions through their development, and then influence the other system. The TTUHTID Coupling System also produces coupling with other external bodies including government (government support (gover) is an important part of the scientific and technological innovation system and promotes scientific and technological innovation by formulating policies, supporting funds, government procurement, innovation subsidies, and tax incentives) [60], sci-tech intermediaries (development of sci-tech intermediary (interme) integrates information and resources based on their intersecting position between supply and demand, which can help high-tech industries identify nonredundant information, expand resource acquisition channels, and promote the introduction, transfer, and diffusion of regional scientific and technological achievements) [61], sci-tech finance (sci-tech finance (fina) is an important part of the national sci-tech innovation system and financial system) [62, 63], and other research institutions (other research institutions (insti) correspond to the "research" in the sci-tech innovation system, which is an important part of the system) [64]. These bodies form an integrated sci-tech innovation system of "government-industry-university-research-financeintermediary."

2.5. The Coupling Mechanism between TTU and HTID. Coupling, which originates from physical science, is a phenomenon in which two or more systems influence each other through various interactions [65]. In this paper, both TTU and HTID are regarded as open systems. These two systems interact, penetrate, and couple to TTU-HTID coupling system with new structure and functions. The coupling mechanism is shown in Figure 1.

\section{Research Method and Model}

\subsection{Comprehensive Evaluation Index}

3.1.1. Evaluation Index System. According to related references [66-69], the evaluation index systems for the ability of TTU and the level of HTID are determined, respectively, as shown in Tables 1 and 2 . 


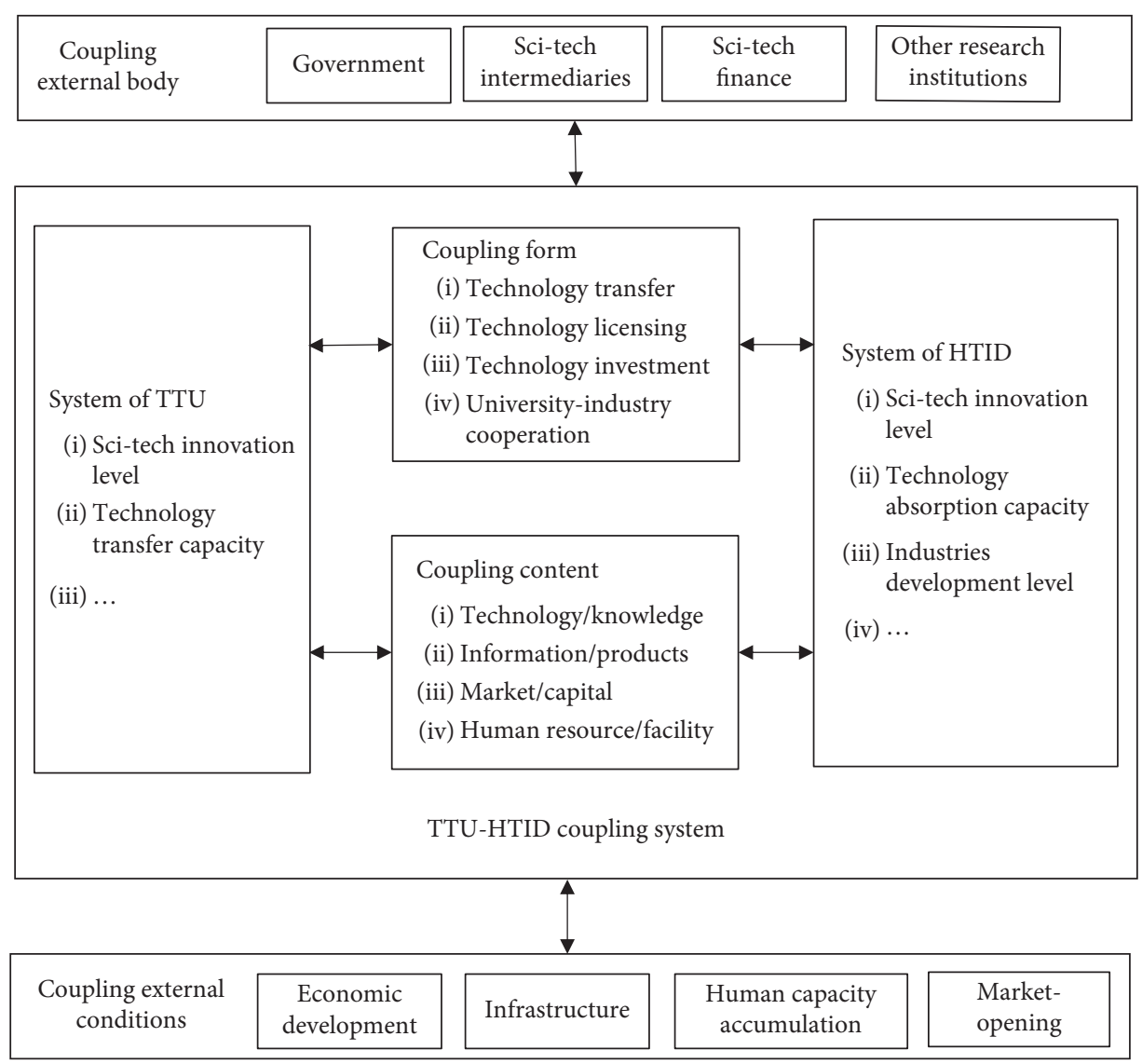

Figure 1: TTU-HTID coupling system.

TABLE 1: Evaluation index system of ability of TTU.

\begin{tabular}{ll}
\hline First-level index & Second-level index \\
& (i) Number of colleges \\
& (ii) Research and development staff \\
& (iii) R\&D achievement application and technical service personnel \\
& (iv) Research and development project funding/thousand yuan \\
& (v) R\&D achievement application and technology service project funding/thousand yuan \\
(i) Conditions and basis & (vi) Research and development institution \\
& (vii) Research and development project \\
& (viii) R\&D achievement application and technology service project \\
& (ix) Published scientific and technological work \\
& (x) Published academic paper \\
& (xi) Number of patents granted \\
(ii) Scientific and technological achievements The number of national awards won by the achievements & (xii) Ther \\
& (xiii) Number of signed contracts for technology transfer \\
& (xiv) Actual income in the year of technology transfer/thousand yuan \\
\hline (iii) Transfer effect &
\end{tabular}

TABLE 2: Evaluation index system of level of HTID.

First-level index

(i) Scale of development

(ii) Innovation ability
Second-level index

(i) Number of companies

(ii) Average number of employees

(iii) Investment/100 million yuan

(iv) Main business income/100 million yuan

(v) R\&D personnel equivalent to full-time equivalent/person-year

(vi) Internal expenditure of $R \& D$ funds $/ 10,000$ yuan

(vii) New product sale revenue/10,000 yuan

(viii) Number of valid invention patents 
3.1.2. Index Screening. Considering that there is multicollinearity between the second-level indicators in Tables 2 and 3 , it is necessary to screen the indicators. This paper uses the information contribution rate to filter and assign weights to the indicators. This is because indicators screening and weighting based on the information contribution rate can overcome the factor analysis method that does not take into account the distortion of the comprehensive evaluation results due to the high overlap rate of information between the indicators. Therefore, screening indicators based on the information contribution rate can reduce information overlap between evaluation indicators and ensure a scientific and reasonable evaluation indicator system [70]. The calculation steps are as follows:

(1) Calculate the eigenvalues $\lambda_{j}(j=1,2, \ldots, m)$ of $m$ indexes correlation coefficient matrix $X^{T} X$ :

$$
\left|X^{T} X-\lambda_{j} E_{m}\right|=0 .
$$

In equation (1), $X$ is the index data matrix standardized by $Z$ and $E_{m}$ denotes the unit matrix of order $m$.

(2) Determine the key factors to be retained. If the cumulative variance contribution rate $\Omega_{p}$ of $p$ factors with larger variance contribution rate $\omega_{j}$ satisfies the following:

$$
\Omega_{p}=\sum_{j=1}^{P} \omega_{j}=\sum_{j=1}^{P} \frac{\lambda_{j}}{m}>M_{0} .
$$

Then retain these $p$ key factors.

In equation (2), $M_{0}$ takes the larger value $90 \%$.

(3) Calculate factor loading matrix $A=\left(a_{i j}\right)_{m \times p}$ :

$$
A=\left(\lambda_{1}^{1 / 2} \xi_{1}, \lambda_{2}^{1 / 2} \xi_{p}, \ldots, \lambda_{p}^{1 / 2} \xi_{p}\right) .
$$

In equation (3), $\xi_{j}(j=1,2, \ldots, p)$ is the eigenvector corresponding to the $j$ th eigenvalue $\lambda_{j}$ of the index set correlation coefficient matrix.

(4) Calculate the information contribution rate $I_{i}$ of the index $X_{i}$, that is, calculate the ratio of the index $X_{i}$ to explain the information contained in the original index set.

$$
I_{i}=\sum_{j=1}^{P} I_{i j}=\sum_{j=1}^{P} \omega_{j}\left(\frac{a_{i j}^{2}}{\sum_{j=1}^{m} a_{i j}^{2}}\right) .
$$

(5) Calculate the cumulative information contribution rate $R_{s}$.

$$
R_{s}=\frac{\sum_{i=1}^{s} I_{m i}}{\sum_{i=1}^{m} I_{i}}
$$

In equation (5), $I_{m i}$ is the information contribution rate of the $i$-th largest index after all $m$ indicators are sorted from large to small.
(6) Screen indicators with high information contribution rate. If the relative cumulative information contribution rate $R_{s}$ satisfies: $R_{s-1}<R_{0} \leq R_{s}$ $\left(R_{0}=0.7\right)$, then $s$ indexes with a larger information contribution rate are reserved.

(7) Check the necessity of eliminating information overlap indicators. If the ill-conditioned index of the remaining $t$ indexes is not greater than 10 , it means that the overall information overlap level of the remaining indexes is not high, and there is no need to eliminate the information overlap indicators; otherwise, it means that the overall information overlap level of the remaining indexes is high. One step further eliminates some of the information overlap indicators and reduces the overall information overlap level between indexes. The ill-conditioned index $\mathrm{CI}_{t}$ can be determined according to the following equation:

$$
\mathrm{CI}_{t}=\sqrt{\frac{\lambda_{1}^{*}}{\lambda_{t}^{*}}}
$$

In equation (6), $\lambda_{1}^{*}$ and $\lambda_{t}^{*}$ are the maximum and minimum eigenvalues of $t$ index correlation coefficient matrices, respectively.

(8) Eliminate indicators with a high degree of information overlap. The index with the relatively small information contribution rate is eliminated from the two largest absolute value of the Person correlation coefficient among the remaining $t$ indexes. Then, the step (7) and (8) are repeated and iterate over the remaining indexes, until the ill-conditioned index of the remaining $k$ indicators $\mathrm{CI}_{k}$ is not greater than 10 , the elimination of the information overlap indicators is stopped.

(9) Determine the weight of the evaluation index. Assuming that the final retained indicators after indicator screening are $X_{1}^{*}, X_{2}^{*}, \ldots, X_{k}^{*}$, and the corresponding information contribution rates are $I_{1}^{*}, I_{2}^{*}, \ldots, I_{k}^{*}$, according to the normalization method, the weight $\omega_{u}$ of the available index $X_{u}^{*}(1 \leq u \leq k)$ is as follows:

$$
\omega_{u}=\frac{I_{u}^{*}}{\sum_{j=1}^{k} I_{j}^{*}} .
$$

According to the above algorithm, the evaluation index systems for the ability of TTU and the level of HTID are shown in Tables 3 and 4.

3.1.3. Determining the Comprehensive Evaluation Index. According to Tables 3 and 4, the comprehensive evaluation index of the ability of TTU is calculated as follows:

$$
U_{1}=\sum_{i=1}^{n} u 1_{i} \omega 1_{i}
$$


TABLE 3: Evaluation index system of ability of TTU after screening.

\begin{tabular}{ll}
\hline Screened indexes & Weight \\
\hline (i) Published scientific and technological work & 0.3418 \\
(ii) Actual income in the year of technology transfer/thousand yuan & 0.2597 \\
(iii) Number of signed contracts for technology transfer & 0.2108 \\
(iv) Number of patents granted & 0.1877 \\
\hline
\end{tabular}

TABLE 4: Evaluation index system of level of HTID after screening.

\begin{tabular}{lr}
\hline Screened indexes & Weight \\
\hline (i) Average number of employees & 0.2907 \\
(ii) Number of companies & 0.1918 \\
(iii) R\&D personnel equivalent to full-time equivalent/person-year & 0.1811 \\
(iv) Investment/100 million yuan & 0.1766 \\
(v) Internal expenditure of R\&D funds/10,000 yuan & 0.1598 \\
\hline
\end{tabular}

The comprehensive evaluation index of the level of HTID is calculated as follows:

$$
U_{2}=\sum_{j=1}^{m} u 2_{j} \omega 2_{j}
$$

In the above two equations, $u 1$ and $u 2$ are the standardized numerical values of the evaluation indexes for the ability of TTU and the level of HTID, respectively, and $\omega 1$ and $\omega 2$ are the corresponding weights of the indexes.

3.2. Coupling Coordination Index. The coupling index (CI) describes the mutual influence between two or more systems themselves and external factors [71]. This article uses the CI model to describe the relationship between the ability of TTU and the level of HTID. Following Wang and Wang [72], the CI is as follows:

$$
C=\left[\frac{U_{1} \times U_{2}}{\left(\left(U_{1}+U_{2}\right) / 2\right)^{2}}\right]^{1 / 2} .
$$

According to equation (10), coupling index $C \in[0,1]$. When $C=1$, TTU and HTID are resonance coupling; when $C=0$, there is no coupling between TTU and HTID.

Although CI can reflect the strength of the interaction between the ability of TTU and the level of HTID, it is not enough to fully reflect coordination between them. When comparing and analyzing the ability of TTU and the level of HTID in different provinces, the CI between the ability of TTU and the level of HTID may be the same as that of provinces with lower levels. Relying solely on the CI cannot effectively present the regional differences between the ability of TTU and the level of HTID. Therefore, based on the CI function, the coupling coordination index (CCI) model is proposed as follows:

$$
\begin{aligned}
& D=\sqrt{C \times T}, \\
& T=\alpha U_{1}+\beta U_{2} .
\end{aligned}
$$

$D$ is CCI of the ability of TTU and the level of HTID (TTUHTID CCI), $T$ is the comprehensive coordination index of them, $\alpha$ and $\beta$ are undetermined coefficients that depend on the importance of each subsystem in the system. Because TTU and HTID both have a significant impact on the coupling system, it is set $\alpha=0.5$ and $\beta=0.5$.

Research on quantitative evaluation of CCI with related literature [73], TTU-HTID CCI is divided into 4 levels from low to high to intuitively reflect the coupling coordination between them. The classification criteria are shown in Table 5.

3.3. Productivity Index. CI and CCI are both measured values jointly displayed by TTU and HTID. They are a measure of the close relationship between TTU and HTID and the level of coupling development which cannot indicate the comparative relationship between TTU and HTID. To reflect the difference between TTU and HTID, the concept of productivity index (PI) is proposed. TTU PI refers to the ratio of the ability of TTU and the level of HTID, which is a measure of the degree of advancement or lag in TTU relative to the HTID. The formula can be expressed as follows:

$$
P=\frac{U_{1}}{U_{2}}
$$

In equation (12), $P$ on behalf of TTU PI, when $P<1, U_{1}$ lags the $U_{2}$, when $P=1, U_{1}$ is equivalent to $U_{2}$, when $P>1$, $U_{1}$ is ahead of $U_{2}$.

3.4. Rank Correlation Coefficient between CCI and PI. To better show the time series characteristics of TTU-HTID CCI and TTU PI in each province, the Spearman rank correlation coefficient method was used to analyze the trend of changes in related indicators from 2010 to 2019. The calculation formula of the rank correlation coefficient is shown as follows:

$$
R_{n}=1-\left[\frac{6 \cdot \sum_{i=1}^{n}\left(X_{i}-Y_{i}\right)^{2}}{N^{3}-N}\right] .
$$

In equation (13), $R_{n}$ is rank correlation coefficient, $X_{i}$ is the serial number from 2010 to 2019 in descending order of evaluation value, $Y_{i}$ is the serial number arranged by time, and $N$ is the number of samples. 
TABle 5: Classification standard of CCI.

\begin{tabular}{lc}
\hline CCI & Coupling coordination level \\
\hline$[0,0.3)$ & Low coupling coordination $(\mathrm{L})$ \\
{$[0.3,0.5)$} & Moderately coupling coordination $(\mathrm{M})$ \\
{$[0.5,0.8)$} & Highly coupling coordination $(\mathrm{H})$ \\
{$[0.8,1]$} & Extremely coupling coordination $(\mathrm{E})$ \\
\hline
\end{tabular}

The absolute value of $R_{n}$ is compared with the critical value $W_{P}$ in the rank correlation coefficient statistics table. If $\left|R_{n}\right| \geq W_{P}$, it indicates that the changing trend is significant. When $R_{n}$ is positive, it shows an upward trend, and when $R_{n}$ is negative, it shows a downward trend. Therefore, when the $R_{n}$ of TTU-HTID CCI is positive, it means that CCI displays an upward trend and CCI is increasing; otherwise, it is showing a downward trend and CCI is decreasing. When the $R_{n}$ of TTU PI is positive, it indicates an upward trend for PI; otherwise, it shows a downward trend.
3.5. Influencing Factor Measurement Mode. According to TTU-HTID Coupling System (shown as Figure 1), in addition to TTU and HTID, the TTU-HTID coupling system also includes external entities such as government, sci-tech intermediaries, sci-tech finance, other research institutions, and other external subjects and environment which may also affect the TTU-HTID coupling coordination. Considering the influence of the external environment is multifaceted and invisible, which is easy to mix with other factors, this study only analyzes the influence of the internal subjects of the sci-tech innovation system "government, industry, university, research, and financial institutions" on TTUHTID CCI, except for external environmental factors.

Table 6 shows the summary of influencing factors and the corresponding evaluation index.

Based on the above analysis of influencing factors, the following measurement model is established:

$$
\begin{aligned}
C_{i, t}= & \beta_{0}+\beta_{1} \ln \text { Utech }_{i, t}+\beta_{2} T T A U_{i, t}+\beta_{3} \ln \text { Htech }_{i, t}+\beta_{4} \ln \text { Habsorb }_{i, t}+\beta_{5} H T I D L_{i, t}+\beta_{6} \ln \text { cooper }_{i, t}+\beta_{7} \ln \text { gover }_{i, t} \\
& +\beta_{8} \ln \text { interme }_{i, t}+\beta_{9} \ln \text { fina }_{i, t}+\beta_{10} \ln \text { insti }_{i, t}+\varepsilon_{i, t} .
\end{aligned}
$$

In equation (14), $i$ and $t$ represent province and year, respectively; $C$ is explained variable and TTU-HTID CCI whose data is calculated from the above; $\beta_{0}$ is constant term; $\varepsilon$ represents the random error term. Considering the large values of some explanatory variables, to facilitate analysis, these variables are processed in logarithm.

Since TTU-HTID CCI has a reverse causal relationship to the related influencing factors of TTU and HTID, to overcome the possible endogenous problems, SYS-GMM is used to estimate the measurement model. In the specific estimation process, the three-period lags of explanatory variables are selected as instrumental variables.

3.6. Data Selection. Panel data of the TTU and the HTID in 31 provinces in Mainland China from 2010 to 2019 is selected for empirical analysis (Due to the limitation of data collection, Hong Kong, Macao, and Taiwan regions are not included, the same below). Data sources include "Compilation of Statistics on Science and Technology of Higher Education Institutions," "Statistical Yearbook of China's High-tech Industry," "Yearbook of Statistics of China's High-tech Industry," etc. Some of the data of HTID in 2017 are missing, used by the linear interpolation method to complete [66]. To eliminate the influence of different magnitudes and dimensions among various indicators and make the data comparable, the original data of each indicator is processed without dimension.

\section{Results and Discussion}

\subsection{Coupling and Coordination Analysis}

4.1.1. Analysis of Each Region. Based on the comprehensive evaluation of TTU and HTID in 31 provinces in China from 2010 to 2019, the average value of TTU-HTID CCI and TTU PI in each year of the country and the four major regions are calculated using the CCI and PI model, shown in Figure 2.

It can be seen from Figure 2 that from 2010 to 2019, the national TTU-HTID CCI showed a gradual upward trend, and the average value reached the moderate coupling coordination level in 2016; the TTU PI was fluctuating and decreasing, after reaching the lowest value of 3.118 in 2015 there has been an increase again, indicating that the growth rate of the level of HTID is higher than the ability of TTU, but the ability of TTU is much higher than the level of HTID, the conversion efforts of scientific and technological achievements of universities into high-tech industries still needs to be improved.

Related policies successively released in 2015-2016 have further promoted the transformation and development of TTU, stimulated the vitality of the transformation of TTU, and made the PI of the transformation of TTU show an upward trend after 2016. In terms of subregions, in the past five years, the TTU-HTID CCI shows East > Central > Northeast > West, and TTU PI shows the spatial distribution characteristics of Central $<$ East $<$ West $<$ Northeast. The specific analysis of the four major regions is as follows: 
TABLE 6: Influencing factors and evaluation index.

\begin{tabular}{lc}
\hline Influencing factor & Evaluation index \\
\hline Utech & \\
cooper & Internal expenditure of university R\&D funds \\
Habsorb & Enterprise funds in university R\&D funds \\
Htech & Cost of technological transformation of the high-tech industry \\
gover & Internal expenditure of R\&D funds in high-tech industry \\
interme & Government funds in the internal expenditures of R\&D \\
fina & Technical market turnover \\
insti & Other funds in the internal expenditures of R\&D expenditures \\
\hline
\end{tabular}

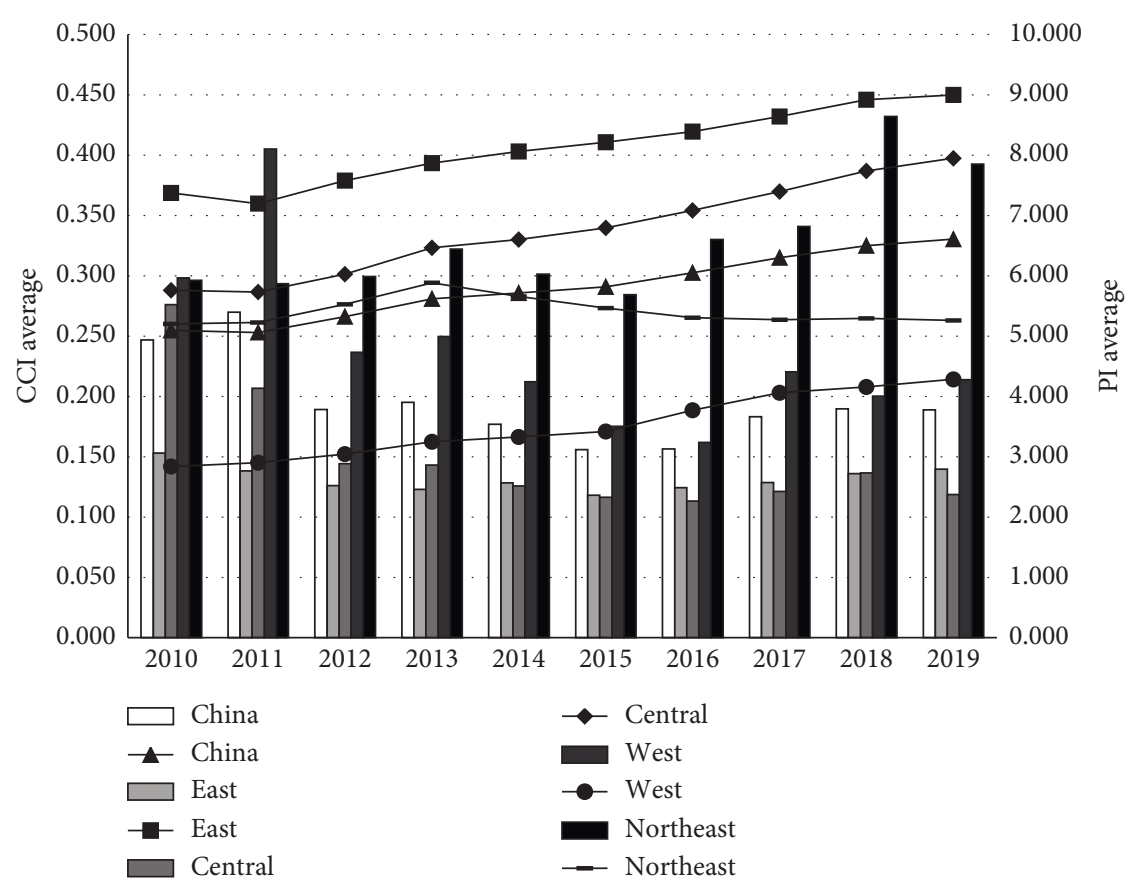

FIgure 2: 2010-2019 Chinese and four major regions TTU-HTID CCI and TTU PI average.

(1) East Region. The TTU-HTID CCI is the highest, which is much higher than the national average, showing an upward trend year by year. It exceeded 0.3 in 2010, reaching the level of moderately coupling coordination, and reached 0.450 in 2019, approaching highly coupling coordination. This suggests that the interactive relationship between TTU and HTID tends to be close and the development is in a good stage. TTU PI is lower than the national average level, showing a state of declining volatility, reaching the lowest value of 2.359 in 2015, indicating that although the ability of the TUU comprehensive evaluation index is ahead of the level of HTID, the ability of TUU and the level of HTID tend to be balanced and can be developed relatively coordinated.

(2) Central Region. The TTU-HTID CCI is slightly higher than the national average, and it is also showing an upward trend year by year. It was at low coupling coordination in 2010 and has reached moderate coupling coordination since 2012, which shows the interactive relationship between TTU and HTID is gradually improving, representing a healthy state of development. TTU PI was higher than the national average in 2010, and then the downward trend was more obvious. It has reached the lowest level in the country since 2014, but it is still greater than 2. It shows that the comprehensive evaluation index of the ability of TTU is also ahead of the level of HTID, and the ability of TTU and the level of HTID tend to be balanced.

(3) West Region. The TTU-HTID CCI is far lower than the national average, but it still shows an upward trend year by year. The CCI in 2019 was 0.214 , which is still at low coupling coordination, showing that the interaction between TTU and HTID is gradually improving. However, the mutual coupling and coordination are at a low level, as well as the development situation. TTU PI is higher than the national average level and shows a state of declining fluctuations, reaching the lowest value of 3.235 in 2016, indicating that the TUU level is ahead of the HTID. 
Considering TTU and HTID are in a state of low coupling coordination, it shows that they cannot support each other well. TUU's support for HTID is not enough, and the HTID foundation is poor.

(4) Northeast Region. The TTU-HTID CCI was slightly higher than the national average before 2013 and was in a slow-growth state; after 2014, it gradually declined and was lower than the national average, and the CCI was always below 0.3 , at low coupling coordination, which shows that the interaction between TTU and HTID is gradually getting worse, and mutual support weakens. In recent years, TTU PI is much higher than the national average. Combining the CCI between TTU and HTID shows that the ability of TUU has increased to a certain extent, but it has not been transformed into the driving force of HTID, which is in a state of shrinking.

4.1.2. Analysis of Each Province. Table 7 shows the $R_{n}$ of TTU-HTID CCI and TTU PI in each province from 2010 to 2019 (specific data shown in Table 8: 2010-2019 CCI and PI of each province).

This study collected 10 years of data, $N=10$, to check the critical value of Spearman's rank correlation coefficient $W_{P}=0.564(\alpha=0.05)$. Data greater than 0.564 are indicated by “*” in Table 7 .

It can be seen from Table 7 that, except for the three provinces of Tianjin, Liaoning, and Heilongjiang (indicated by "-" in Table 7), the $R_{n}$ of TTU-HTID CCI in all provinces are positive, indicating that $\mathrm{CCI}$ is on an upward trend, and the upward trend in most provinces is more obvious. The $R_{n}$ of Shanghai, Tibet, and Jilin are lower than the critical value, indicating that the TTU-HTID CCI has not increased significantly.

There are 13 provinces with positive $R_{n}$ of TTU PI, including Beijing, Jiangsu, Zhejiang, Shandong, Guangdong, Hainan, Shanxi, Inner Mongolia, Sichuan, Guizhou, Liaoning, Jilin, and Heilongjiang. Among them, the $R_{n}$ of Jiangsu and Liaoning exceeded the critical value, indicating that PI increased significantly, and the growth rate of the ability of TTU was significantly restored compared with the level of HTID. Totally there are 18 provinces with negative $R_{n}$ (indicated by "-" in Table 7). Among them, the absolute values of $R_{n}$ in Hebei, Anhui, Hubei, Hunan, Chongqing, Qinghai, and Xinjiang, exceed the critical value, indicating that the level of HTID has increased significantly faster than the ability of TTU.

Based on the classification standard of CCI of each province, it can be classified as follows.

Only Jiangsu is at the level of extreme coupling coordination. According to the statistics in 2019, there are a total of 149 universities in Jiangsu, 24,368 patents authorized by universities, and 2,515 technology transfer contracts. The three indexes above all rank the first in China, and other indexes of TTU also rank the top. There are 48 high-tech zones at or above the provincial level, among which 18 are national levels, ranking first in China. The HTI also performs well in various indicators. TTU and HTID both are showed high activity $\left(\left|R_{n}\right| \geq W_{P}\right)$ and good balance (TTUPI = $1.225)$, and CCI shows an outstanding performance.

Provinces with high coupling coordination include Guangdong and Zhejiang, but Guangdong has a much higher CCI. Guangdong has a very outstanding development level of HTI, ranking first in many indicators in China. Its TTU PI is less than 1, but TTU PI Rank Correlation Coefficient is close to $W_{P}$, indicating a significant growth trend of TTU. In the future, the CCI of Guangdong will increase faster, and Zhejiang increases stably.

14 provinces are in the level of moderately coupling coordination, distributed in 4 regions and divided into 4 types:

(i) The first type includes the characteristics with CCI rank correlation coefficient a positive number, TTU PI higher than 1, and TTU PI rank correlation coefficient a negative number (represented as “++-"), including Hebei, Shanghai, Anhui, Hunan, Hubei, Henan, Chongqing, and Shaanxi. CCI of the provinces above is ascending every year, and TTU higher than HTID with the gap narrowing, indicating a good development trend of CCI. Because the absolute value of both rank correlation coefficients is higher than $W_{P}$ in Hebei, Anhui, Hunan, Hubei, and Chongqing, the CCI of the 5 provinces will increase at a faster rate.

(ii) CCI rank correlation coefficient of Fujian and Jiangxi is a positive number, TTU PI lower than 1, and TTU PI rank correlation coefficient a negative number and the absolute value lower than $W_{P}$ (represented as "+--"). It means that the base of TTU is weak and a narrowing gap compared with HTID exists. CCI of the two provinces will have a relative development.

(iii) The indexes of Beijing, Shandong, and Sichuan can be represented as "+++." The ability of TTU is higher than the level of HTID, and the gap existing is not an appreciable expansion. It means there are very rich resources in the three provinces, but not enough to transfer to HTID. Therefore, it should overcome this barrier to guarantee the promotion of CCI.

(iv) The indexes Liaoning can be represented as "-++," which means the ability of TTU is higher than the level of HTID, and the gap is becoming more and more obvious. Most important of all, CCI of Liaoning shows a downward trend. The gradually improved ability of TTU has not brought the development of HTID, and CCI is getting worse and worse.

There are 14 provinces in the level of low coupling coordination, distributed in 4 regions and also divided into 4 types:

(i) The indexes of Hainan, Shanxi, Inner Mongolia, Guizhou, and Jilin can be represented as "+++." The ability of TTU is higher than the level of HTID, and 
TABLe 7: TTU-HTID coupling coordination indexes of each province.

\begin{tabular}{|c|c|c|c|c|c|c|c|c|}
\hline Region & Province & $\begin{array}{l}\text { CCI } \\
2010 \\
\end{array}$ & $\cdots$ & $\begin{array}{l}\text { CCI } \\
2019 \\
\end{array}$ & $\begin{array}{l}\text { Classification standard } \\
2019\end{array}$ & $\begin{array}{c}\text { CCI rank correlation } \\
\text { coefficient }\end{array}$ & $\begin{array}{l}\text { TTU } \\
\text { PI }\end{array}$ & $\begin{array}{c}\text { TTU PI rank correlation } \\
\text { coefficient }\end{array}$ \\
\hline \multirow{10}{*}{ East } & Hainan & 0.075 & $\ldots$ & 0.093 & $\mathrm{~L}$ & $0.661^{*}$ & 5.536 & 0.285 \\
\hline & Tianjin & 0.259 & $\ldots$ & 0.260 & $\mathrm{~L}$ & $-0.309-$ & \multirow{3}{*}{$\begin{array}{c}1.812 \\
2.728 \\
0.966 \\
-\end{array}$} & $-0.261-$ \\
\hline & Hebei & 0.246 & $\ldots$ & 0.323 & M & $0.915^{*}$ & & $-0.576-^{*}$ \\
\hline & Fujian & 0.301 & $\cdots$ & 0.353 & M & $0.927^{*}$ & & $-0.261-$ \\
\hline & Shanghai & 0.379 & $\ldots$ & 0.432 & M & 0.055 & 3.298 & $-0.103-$ \\
\hline & Beijing & 0.410 & $\ldots$ & 0.461 & M & $0.721^{*}$ & 8.768 & 0.042 \\
\hline & Shandong & 0.416 & $\ldots$ & 0.476 & M & $0.842^{*}$ & 1.795 & 0.139 \\
\hline & Zhejiang & 0.403 & $\ldots$ & 0.541 & $\mathrm{H}$ & $0.952^{*}$ & \multirow{2}{*}{$\begin{array}{c}1.367 \\
0.429 \\
-\end{array}$} & 0.212 \\
\hline & Guangdong & 0.572 & $\cdots$ & 0.750 & $\mathrm{H}$ & $0.952^{*}$ & & 0.552 \\
\hline & Jiangsu & 0.627 & $\ldots$ & 0.809 & $\mathrm{E}$ & $0.976^{*}$ & 1.225 & $0.697^{*}$ \\
\hline \multirow{6}{*}{ Central } & Shanxi & 0.189 & $\ldots$ & 0.227 & $\mathrm{~L}$ & $0.891^{*}$ & \multirow{2}{*}{$\begin{array}{c}4.227 \\
0.852 \\
-\end{array}$} & 0.055 \\
\hline & Jiangxi & 0.237 & $\ldots$ & 0.364 & M & $0.988^{*}$ & & $-0.442-$ \\
\hline & Anhui & 0.318 & $\ldots$ & 0.402 & M & $0.721^{*}$ & 1.362 & $-0.952-*$ \\
\hline & Hunan & 0.300 & $\ldots$ & 0.444 & M & $0.976^{*}$ & 2.596 & $-0.855-^{*}$ \\
\hline & Hubei & 0.323 & $\ldots$ & 0.464 & M & $1.000^{*}$ & 2.445 & $-0.733-^{*}$ \\
\hline & Henan & 0.361 & $\ldots$ & 0.483 & M & $0.976^{*}$ & 2.767 & $-0.285-$ \\
\hline \multirow{12}{*}{ West } & Xizang & 0.019 & $\ldots$ & 0.025 & $\mathrm{~L}$ & 0.515 & 3.844 & $-0.248-$ \\
\hline & Qinghai & 0.039 & $\ldots$ & 0.073 & $\mathrm{~L}$ & $0.879^{*}$ & 1.358 & $-0.697-^{*}$ \\
\hline & Ningxia & 0.045 & $\ldots$ & 0.100 & $\mathrm{~L}$ & $0.891^{*}$ & 2.990 & $-0.394-$ \\
\hline & Xinjiang & 0.068 & $\ldots$ & 0.101 & $\mathrm{~L}$ & $0.830^{*}$ & 5.544 & $-0.685-^{*}$ \\
\hline & Gansu & 0.127 & $\ldots$ & 0.161 & $\mathrm{~L}$ & $0.879^{*}$ & 8.111 & $-0.297-$ \\
\hline & $\begin{array}{c}\text { Inner } \\
\text { Mongolia }\end{array}$ & 0.126 & $\ldots$ & 0.177 & $\mathrm{~L}$ & $0.952^{*}$ & 7.160 & 0.285 \\
\hline & Yunnan & 0.133 & $\ldots$ & 0.213 & $\mathrm{~L}$ & $0.939^{*}$ & 6.575 & $-0.491-$ \\
\hline & Guangxi & 0.173 & $\ldots$ & 0.216 & $\mathrm{~L}$ & $0.927^{*}$ & 1.802 & $-0.188-$ \\
\hline & Guizhou & 0.129 & $\ldots$ & 0.224 & $\mathrm{~L}$ & $0.964^{*}$ & 3.159 & 0.515 \\
\hline & Chongqing & 0.233 & $\ldots$ & 0.398 & M & $0.988^{*}$ & 3.301 & $-0.612-^{*}$ \\
\hline & Shaanxi & 0.297 & $\ldots$ & 0.418 & M & $1.000^{*}$ & 4.666 & $-0.382-$ \\
\hline & Sichuan & 0.315 & $\ldots$ & 0.465 & M & $0.964^{*}$ & 2.796 & 0.188 \\
\hline \multirow{3}{*}{ Northeast } & Heilongjiang & 0.229 & $\ldots$ & 0.232 & $\mathrm{~L}$ & $-0.103-$ & 9.008 & 0.309 \\
\hline & Jilin & 0.216 & $\ldots$ & 0.233 & $\mathrm{~L}$ & 0.297 & 5.205 & 0.285 \\
\hline & Liaoning & 0.336 & $\ldots$ & 0.323 & M & $-0.661-^{*}$ & 9.337 & $0.697^{*}$ \\
\hline
\end{tabular}

the gap existing is not an appreciable expansion. Combined with the low indexes of HTID and insufficient ability of TTU in these provinces, the development of CCI will be slow.

(ii) The indexes of Tianjin can be represented as “-+-." It means CCI of Tianjin is going down, and the ability of TTU is higher than the level of HTID, but the gap is becoming close. Both the ability of TTU and the level of HTID are shrinking.

(iii) It can be represented as “++-" of the indexes of Xizang, Qinghai, Ningxia, Xinjiang, Gansu, Yunnan, and Guangxi. Given the weak foundation for development, the growth of CCI will be relatively slow. Because the absolute value of both rank correlation coefficients is higher than $W_{P}$ in Qinghai and Xinjiang, CCI will increase at a faster rate.

(iv) The indexes Heilongjiang can be represented as “-++," which is same to Liaoning. Considering its poorer foundation, CCI of Heilongjiang will be getting worse and worse.
4.2. Influencing Factors of Coupling Coordination of TTU and HTID. Since the TTU-HTID coupling coordination degree has a reverse causal relationship for the transformation of university scientific and technological achievements and the development of high-tech industry related factors, in order to overcome the possible endogenous problems, the system GMM method is used to estimate the measurement model. In the specific estimation process, the three-period lag of the explanatory variable is selected as the instrumental variable, and the measurement results are shown in Table 9. It can be seen from Table 9 that the indicators of the Arellano-Bond test, Sargan test, and Hansen test meet the requirements, and the number of instrumental variables does not exceed the number of endogenous variables, and they are all valid, indicating that the system GMM estimation results are effective.

From SYS-GMM estimation results, it can be seen that the influencing factors of the HTID subsystem have a significant impact on the TTU-HTID CCI, but Utech, TTAU in the TTU subsystem have no significant influence. Htech, Habsorb, HTIDL all have significant promoting effects on 


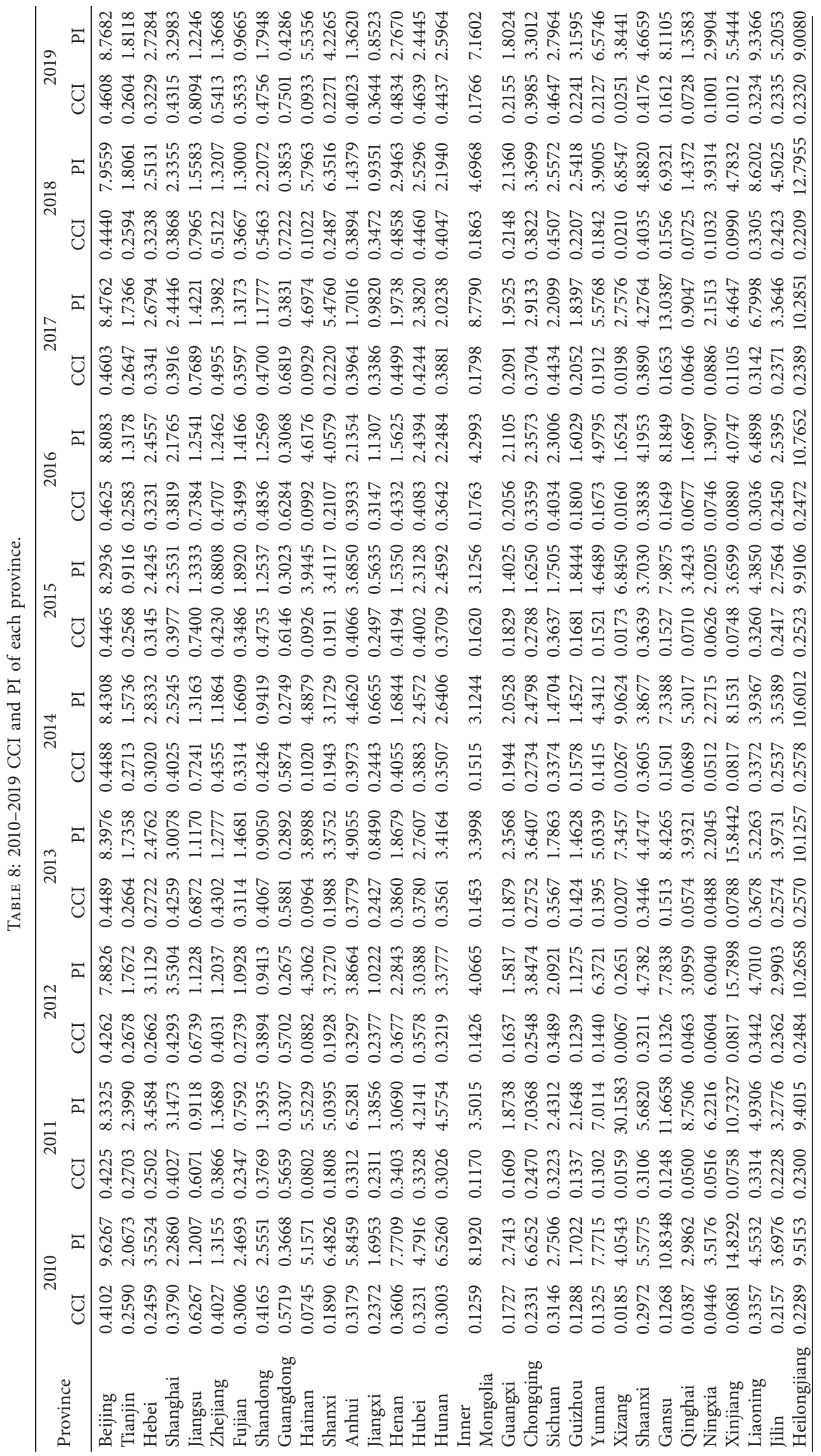


TABLE 9: Influencing factors of TTU-HTID coupling coordination by SYS-GMM.

\begin{tabular}{|c|c|c|}
\hline Variable & Coefficient & $P$ value \\
\hline lnUtech & 0.014 & 0.242 \\
\hline TTAU & 0.156 & 0.133 \\
\hline InHtech & $0.014^{* *}$ & 0.015 \\
\hline lnHabsorb & $0.006^{* * *}$ & 0.008 \\
\hline HTIDL & $0.504^{* * *}$ & 0.000 \\
\hline Incooper & $0.012^{* *}$ & 0.021 \\
\hline lngover & -0.037 & 0.104 \\
\hline Lninterme & -0.001 & 0.922 \\
\hline $\operatorname{lnfina}$ & $0.014^{* * *}$ & 0.005 \\
\hline lninsti & 0.030 & 0.052 \\
\hline _cons & $-0.344^{* * *}$ & 0.001 \\
\hline$P$ & \multicolumn{2}{|c|}{$\leq 0.001$} \\
\hline$A R(1)$ & \multicolumn{2}{|c|}{0.035} \\
\hline$A R(2)$ & \multicolumn{2}{|c|}{0.512} \\
\hline Sargan & \multicolumn{2}{|c|}{0.083} \\
\hline Hansen & \multicolumn{2}{|c|}{0.449} \\
\hline
\end{tabular}

Note. The symbols ${ }^{* * *}$ and ${ }^{* *}$ indicate significant at $1 \%$ and $5 \%$, respectively.

the CCI, whose influence coefficients are 0.014, 0.006, 0.504 . The reason is that from the perspective of national and regional TTU PI, TTU is much higher than HTID, and the improvement of TTU-HTID CCI is more dependent on HTID. Because the evaluation of Utech and TTAU is based on the quantity index, the patent quality, which represents the ability of sci-tech achievements of universities, does not increase significantly with the increase of quantity [74]. However, sci-tech achievements with high quality are more likely to be transferred to HTI. It should improve quality rather than quantity to promote the ability of sci-tech achievements of universities. As an important channel for the technology transfer from universities to HTI, cooper has a significant impact on CCI.

Of all the external entities, only fina has a significant impact on CCI, but gover, interme, and insti have no significant influence. Innovation requires more than technology. It also needs capital to commercialize it, hence, the need for customers to develop connectivity with the capital markets [75]. Technology finance provides financial support for TTU and HTID through a variety of systems such as the four major financial systems represented by banks, guarantees, insurance, and bonds, which is the lifeblood of TTU and HTID. The reason why gover has no significant influence on CCI may be in the following: the vast majority of the government funds to universities are invested in basic research and experimental development research rather than technology transfer. The expected results of the funding are more about the cultivation of students, the publication of articles, the acquisition of patent authorization, etc., and there are few indicators of technology transfer. interme represented by the technology market has a low degree of development and low added value of technology. According to the Statistical Yearbook of China Science and Technology, as the seller in 2019, the value of contract deals in technical markets of high education is 59 million yuan, which is only $2.6 \%$ of the whole market. Moreover, the proportion has been decreasing year by year (from $4.6 \%$ in 2012 to $2.6 \%$ in
2019), which means interme has failed to build a good bridge between universities and HTI. Although insti is in a competitive and cooperative position with universities in the industry-university-research system, they have their own advantages, and the development degree of insti does not have a significant impact on the coupling.

\section{Conclusion and Enlightenment}

5.1. Main Conclusions. This paper focuses on coupling coordination between TTU and HTID in China, analyzes the coupling mechanism of TTU and HTID, and constructs the tailored coupling system for them and based on the conclusions obtained can provide decision-makers with decision-making ideas. The methods adopted here are based on the information contribution rate to screen the evaluation indicators of the two subsystems and assign weights. Two comprehensive evaluation index systems are established and TTU-HTID CI, TTU-HTID CCI, and TTU PI models are introduced as well. Furthermore, the spatiotemporal characteristics of the coupling and coordination of TTU and HTID from 2010 to 2019 are analyzed and the SYS-GMM is applied for estimating its influencing factors. The main conclusions derived from this paper are summarized as follows:

(1) The overall national TTU-HTID CCI shows a gradual upward trend, but the level of coupling coordination is not high. From a regional perspective, TTU-HTID CCI East $>$ Central > Northeast > West, the CCI of Northeast showed a trend of first rising and then falling; TTU PI showed the spatial distribution characteristics of Central $<$ East $<$ West $<$ Northeast. Affected by various factors, the TTU-HTID CCI difference among provinces in each region is East $>$ West $>$ Central $>$ Northeast, and the TTUHTID CCI difference of each province shows a gradually expanding trend.

(2) The national TTU PI is in a state of volatility and decline, and it has risen after reaching the lowest value of 3.118 in 2015, which shows the growth rate of the level of HTID is higher than the ability of TTU, but the ability of TTU is still much higher than the level of HTID. The transformation of Technology in universities into high-tech industries still needs to be improved.

(3) Specific to each province, TTU-HTID CCI has large regional differences, showing a trend of high in the east and low in the west, high in the coast, and low in the inland. Except for Tianjin and Hainan in the east, the TTU-HTID CCI levels of all provinces are relatively high, and the CCI of Jiangsu, Guangdong, and Zhejiang have reached highly coupling coordination. Apart from Shanxi Province in the central, all provinces TTU-HTID CCI have reached moderately coupling coordination. The TTU-HTID CCI of all provinces in the west is generally low, but Sichuan, Shaanxi, and Chongqing have reached moderately coupling coordination. Heilongjiang and Jilin in the 
northeast are at low coupling coordination, while Liaoning has been at moderately coupling coordination, but CCI has not increased significantly.

(4) Except for Tianjin, Liaoning, and Heilongjiang, the TTU-HTID CCI of other provinces is on an upward trend which is obvious in most provinces. TTU PI in 13 provinces is in a state of growth, of which Jiangsu and Liaoning provinces have a significant growth in TTU PI, and the ability of TTU has increased significantly faster than the level of HTID. The TTU PI of 18 provinces is in a state of decline. Among them, Hebei, Anhui, Hubei, Hunan, Chongqing, Qinghai, and Xinjiang have a significant decline in TTU PI, and the level of HTID is significantly faster than the growth rate of the level of TTU.

(5) Htech, Habsorb, HTIDL, cooper, and fina have a significant role in promoting the coupling coordination of TTU-HTID. Utech, TTAU, gover, interme, and insti have no significant impact on TTU-HTID coupling coordination.

\subsection{Policy Enlightenment.}

(1) Based on the regional university resources and industries development, each region should highlight its characteristics, exploit the potential of coupling coordination between TTU and HTID, expand the ability to connect with innovative resources outside the region, and build a multiparticipant industryuniversity-research collaborative innovation platform. Besides, they should also change the unbalanced development of TTU-HTID coupling coordination and reduce the difference of regional TTU-HTID coupling coordination.

(2) All regions should focus on guiding universities to actively integrate into the regional technological innovation system, serving the regional technological innovation chain and industrial chain, and aiming at the common key technologies and cuttingedge leading technologies for industrial development, playing the role of "complement chain," "strong chain," and "chain extension." It is necessary to start from the source of innovation, transform technological achievements into high-tech industries, guide universities and industries to "go out" and "bring in," solve the difficult problem of industrialization of Technology in University, and promote the coupling coordination development of them.

(3) All regions should pay more attention to building a technological innovation system with enterprises as the main body, focus on improving Htech and Habsorb, vigorously broaden the channels for industry-university cooperation, and further strengthen the construction of the technology service system, especially the technology financial system, and give full play to the role of finance in promoting HTID and TTU.

\section{Data Availability}

The data used to support the findings of this study are available from the corresponding author upon request.

\section{Conflicts of Interest}

The authors declare no conflicts of interest.

\section{Authors' Contributions}

FH and LX conceived and designed the research. YQ put forward method and modified the manuscript. FH and WJ drafted the manuscript, prepared figures, and revised the manuscript. All authors have read and agreed to the published version of the manuscript.

\section{Acknowledgments}

This research was funded by the 2020 Social Science Foundation Key Project of Hubei Province (New Think Tank Project) (no. BSKZD2020002) and 2020 Universities Practical Education Project of Hubei Province (no. 2020SJJPE3004).

\section{References}

[1] L. H. Hoc and N. D. Trong, "University-industry linkages in promoting technology transfer: a study of Vietnamese technical and engineering universities," Science, Technology and Society, vol. 24, no. 1, pp. 73-100, 2019.

[2] L. Frolund, F. Murray, and M. Riedel, "Developing successful strategic partnerships with universities," MIT Sloan Management Review, vol. 59, no. 2, pp. 71-79, 2018.

[3] T. Abbate, F. Cesaroni, and A. Presenza, "Knowledge transfer from universities to low- and medium-technology industries: evidence from Italian winemakers," The Journal of Technology Transfer, 2020.

[4] M. Meoli, S. Paleari, and S. Vismara, "Completing the technology transfer process: M\&As of science-based IPOs," Small Business Economics, vol. 40, no. 2, pp. 227-248, 2013.

[5] J. Han, "Technology commercialization through sustainable knowledge sharing from university-industry collaborations, with a focus on patent propensity," Sustainability, vol. 9, p. 1808, 2017.

[6] G. Calcagnini and I. Favaretto, "Models of university technology transfer: analyses and policies," The Journal of Technology Transfer, vol. 41, no. 4, pp. 655-660, 2016.

[7] F. Munari, M. Sobrero, and L. Toschi, "Financing technology transfer: assessment of university-oriented proof-of-concept programmes," Technology Analysis \& Strategic Management, vol. 29, no. 2, pp. 233-246, 2017.

[8] B. Bigliardi, F. Galati, G. Marolla, and C. Verbano, "Factors affecting technology transfer offices' performance in the Italian food context," Technology Analysis \& Strategic Management, vol. 27, no. 4, pp. 361-384, 2015.

[9] G. D. Markman, P. H. Phan, D. B. Balkin, and P. T. Gianiodis, "Entrepreneurship and university-based technology transfer," Journal of Business Venturing, vol. 20, no. 2, pp. 241-263, 2005.

[10] B. N. Sampat, "Patenting and US academic research in the 20th century: the world before and after Bayh-Dole," Research Policy, vol. 35, no. 6, pp. 772-789, 2006. 
[11] M. Kristel, M. A. Maura, and M. A Rodney, "The changing university business model: a stakeholder perspective," R\&D Management, vol. 44, no. 3, pp. 265-287, 2014.

[12] A. Schoen, B. V. de la Potterie, and J. Henkel, "Governance typology of universities' technology transfer processes," Journal of Technology Transfer, vol. 39, no. 3, pp. 435-453, 2014.

[13] A. Muscio, "What drives the university use of technology transfer offices? Evidence from Italy," The Journal of Technology Transfer, vol. 35, no. 2, pp. 181-202, 2010.

[14] A. G. Z. Hu, G. H. Jefferson, and Q. Jinchang, "R\&D and technology transfer: firm-level evidence from Chinese industry," The Review of Economics and Statistics, vol. 87, no. 4, pp. 780-786, 2005.

[15] G. Kruss and M. Visser, "Putting university-industry interaction into perspective: a differentiated view from inside South African universities," The Journal of Technology Transfer, vol. 42, no. 4, pp. 884-908, 2017.

[16] M. Guldbrandsen and J. C. Smeby, "Industry funding and university professors' research performance," Research Policy, vol. 34, no. 6, pp. 932-950, 2005.

[17] S. B. Adams, "Stanford and silicon valley: lessons on becoming a high-tech region," California Management Review, vol. 48, no. 1, pp. 29-51, 2005.

[18] M. Tang, G. S. Walsh, C. Li, and A. Baskaran, "Exploring technology business incubators and their business incubation models: case studies from China," The Journal of Technology Transfer, vol. 46, no. 1, pp. 90-116, 2019.

[19] M. McAdam, K. Miller, and R. McAdam, "University business models in disequilibrium - engaging industry and end users within university technology transfer processes," R\&D Management, vol. 47, no. 3, pp. 458-472, 2017.

[20] L. M. Puerta Sierra, M. E. Marín Vargas, V. G. López Torres, M. Enselmina, L. Torres, and V. Guadalupe, “An institutional framework to explain the university: industry technology transfer in a public university of Mexico," Journal of Technology Management \& Innovation, vol. 12, no. 1, pp. 4-12, 2017.

[21] G. D. Markman, D. S. Siegel, and M. Wright, "Research and technology commercialization," Journal of Management Studies, vol. 45, no. 8, pp. 1401-1423, 2008.

[22] G. Harman, "Australian university research commercialisation: perceptions of technology transfer specialists and science and technology academics," Journal of Higher Education Policy and Management, vol. 32, no. 1, pp. 69-83, 2010.

[23] G. Buenstorf, "Is commercialization good or bad for science? Individual-level evidence from the Max Planck society," Research Policy, vol. 38, no. 2, pp. 281-292, 2009.

[24] O. Al-tabbaa and S. Ankrah, "Social capital to facilitate "engineered" university-industry collaboration for technology transfer: a dynamic perspective," Technological Forecasting and Social Change, vol. 104, pp. 1-15, 2016.

[25] E. Lafuente and J. Berbegal-Mirabent, "Assessing the productivity of technology transfer offices: an analysis of the relevance of aspiration performance and portfolio complexity," The Journal of Technology Transfer, vol. 44, no. 3, pp. 778-801, 2019.

[26] M. Good, M. Knockaert, and B. Soppe, "A typology of technology transfer ecosystems: how structure affects interactions at the science-market divide," The Journal of Technology Transfer, vol. 45, no. 5, pp. 1405-1431, 2020.

[27] M. Grimaldi and L. Cricelli, "Indexes of patent value: a systematic literature review and classification," Knowledge
Management Research \& Practice, vol. 18, no. 2, pp. 214-233, 2020.

[28] L. Lyu, W. Wu, H. Hu, and R. Huang, "An evolving regional innovation network: collaboration among industry, university, and research institution in China's first technology hub," The Journal of Technology Transfer, vol. 44, no. 3, pp. 659-680, 2019.

[29] G. E. Derrick, "Integration versus separation: structure and strategies of the technology transfer office (TTO) in medical research organizations," The Journal of Technology Transfer, vol. 40, no. 1, pp. 105-122, 2015.

[30] J. Blohmke, "Technology complexity, technology transfer mechanisms and sustainable development," Energy for Sustainable Development, vol. 23, pp. 237-246, 2014.

[31] T. J. Soares, A. L. V. Torkomian, and M. S. Nagano, "University regulations, regional development and technology transfer: the case of Brazil," Technological Forecasting and Social Change, vol. 158, Article ID 120129, 2020.

[32] J. Hong, B. Feng, Y. Wu, and L. Wang, "Do government grants promote innovation efficiency in China's high-tech industries?” Technovation, vol. 57-58, no. S1, pp. 4-13, 2016.

[33] B. M. Liang, "Evaluation of high-tech enterprises," Canadian Social Science, vol. 7, no. 6, pp. 245-248, 2011.

[34] S. Ding, "A novel discrete grey multivariable model and its application in forecasting the output value of China's hightech industries," Computers \& Industrial Engineering, vol. 127, pp. 749-760, 2019.

[35] L. Yu, Y. Duan, and T. Fan, "Innovation performance of new products in China's high-technology industry," International Journal of Production Economics, vol. 219, pp. 204-215, 2020.

[36] L. Li, X. Hong, and K. Peng, "A spatial panel analysis of carbon emissions, economic growth and high-technology industry in China," Structural Change and Economic Dynamics, vol. 49, pp. 83-92, 2019.

[37] D. C. Fan and X. M. Gu, "Analysis on the technology innovation efficiency and its influencing factors of Chinese high-tech industry," in Proceedings of the 2018 25th Annual International Conference on Management Science \& Engineering, pp. 149-155, Frankfurt, Germany, 2018.

[38] P. Arora, Q. L. Kweh, and D. Mahajan, "Performance comparison between domestic and international firms in the hightechnology industry," Eurasian Business Review, vol. 8, no. 4, pp. 477-490, 2018.

[39] Y. B. He, S. Zhang, and Z. Y. Zhou, "The pattern of technology diversification and its influence on persistent innovation-a comparative study of China's high technology industries," in Proceedings of the Innovation-driven Capability Building and Industry Development (ISMOT 16), pp. 40-44, Hangzhou, China, 2016.

[40] C. H. Yang, C. C. Kuo, and E. D. Ramstetter, "Intellectual property rights and patenting in China's high-technology industries: does ownership matter?" China World Economy, vol. 19, pp. 102-122, 2011.

[41] M. E. Lovely and Z. Huang, "Foreign direct investment in China's high-technology manufacturing industries," China \& World Economy, vol. 26, no. 5, pp. 104-126, 2018.

[42] J. Cruz-González, P. López-Sáez, J. Emilio Navas-López, and M. Delgado-Verde, "Directions of external knowledge search: investigating their different impact on firm performance in high-technology industries," Journal of Knowledge Management, vol. 18, no. 5, pp. 847-866, 2014.

[43] Y. Zhang, J. Yu, and Y. Liu, "Evolutionary dynamics of high technology industry: modeling of semiconductor sector in 
China," Chinese Management Studies, vol. 7, no. 2, pp. 194-214, 2013.

[44] C. O’Kane, V. Mangematin, W. Geoghegan, and C. Fitzgerald, "University technology transfer offices: the search for identity to build legitimacy," Research Policy, vol. 44, pp. 421-437, 2014.

[45] D. R. D. Moraes Silva, L. O. Lucas, and N. S. Vonortas, "Internal barriers to innovation and university-industry cooperation among technology-based SMEs in Brazil," Industry and Innovation, vol. 27, no. 3, pp. 235-263, 2020.

[46] A. Johnston, "Open innovation and the formation of university-industry links in the food manufacturing and technology sector evidence from the UK," European Journal of Innovation Management, vol. 24, pp. 89-107, 2021.

[47] F. T. Rothaermel and D. L. Deeds, "Alliance type, alliance experience and alliance management capability in hightechnology ventures," Journal of Business Venturing, vol. 21, no. 4, pp. 429-460, 2006.

[48] P. M. A. Paniccia and S. Baiocco, "Co-evolution of the university technology transfer: towards a sustainability-oriented industry: evidence from Italy," Sustainability, vol. 10, pp. 1-29, 2018.

[49] E. Villani, E. Rasmussen, and R. Grimaldi, "How intermediary organizations facilitate university-industry technology transfer: a proximity approach," Technological Forecasting and Social Change, vol. 114, pp. 86-102, 2017.

[50] F. Brescia, G. Colombo, and P. Landoni, "Organizational structures of knowledge transfer offices: an analysis of the world's top-ranked universities," The Journal of Technology Transfer, vol. 41, no. 1, pp. 132-151, 2016.

[51] L. Cassia, A. De Massis, M. Meoli, and T. Minola, "Entrepreneurship research centers around the world: research orientation, knowledge transfer and performance," The Journal of Technology Transfer, vol. 39, pp. 376-392, 2014.

[52] M. Bikard and M. Marx, "Bridging academia and industry: how geographic hubs connect university science and corporate technology," Management Science, vol. 66, no. 8, pp. 3425-3443, 2020.

[53] J. Gorączkowska, "Enterprise innovation in technology incubators and university business incubators in the context of Polish industry," Oeconomia Copernicana, vol. 11, pp. 799$817,2020$.

[54] M. Wagner, "Managing disruptive innovation with technology acquisitions: the informing case of software-based hightechnology industries," Technology Analysis \& Strategic Management, vol. 28, no. 8, pp. 979-991, 2016.

[55] A. D. Daniel and L. Alves, "University-industry technology transfer: the commercialization of university's patents," Knowledge Management Research \& Practice, vol. 18, no. 3, pp. 276-296, 2020.

[56] J. M. Azagra-Caro, R. J. W. Tijssen, and A. Yegros-Yegros, "Measuring macro-level effects of the global economic recession on university-industry research cooperation," in Proceedings of the 21st International Conference on Science and Technology Indicators (STI 2016), Valencia, Spain, 2016.

[57] Y. Moradi and S. Noori, "Entrepreneurial cooperation model between university and SMEs: a case study in Iran," Sustainability, vol. 12, p. 9140, 2020.

[58] O. Leonchuk and D. O. Gray, "Scientific and technological (human) social capital formation and industry-university cooperative research centers: a quasi-experimental evaluation of graduate student outcomes," The Journal of Technology Transfer, vol. 44, no. 5, pp. 1638-1664, 2019.
[59] M. G. Colombo, N. J. Foss, J. Lyngsie, and C. R. Lamastra, "What drives the delegation of innovation decisions? The roles of firm innovation strategy and the nature of external knowledge," Research Policy, vol. 50, Article ID 104134, 2021.

[60] A. Bertello, A. Ferraris, P. De Bernardi, and B. Bertoldi, "Challenges to open innovation in traditional SMEs: an analysis of pre-competitive projects in university-industrygovernment collaboration," International Entrepreneurship and Management Journal, 2021.

[61] A. T. Alexander and D. P. Martin, "Intermediaries for open innovation: a competence-based comparison of knowledge transfer offices practices," Technological Forecasting and Social Change, vol. 80, no. 1, pp. 38-49, 2013.

[62] M. H. Bilgin, L. C. K. Marco, and E. Demir, "Technology transfer, finance channels, and SME performance: new evidence from developing countries," The Singapore Economic Review, vol. 57, Article ID 1250020, 2012.

[63] Z. X. Zhong, "Study about the relationship between scientific and technological finance of emerging industries and economic growth," Agro Food Industry Hi Tech, vol. 28, pp. 1381-1384, 2017.

[64] D. J. Jefferson, M. Maida, A. Farkas, M. Alandete-Saez, and A. B. Bennett, "Technology transfer in the Americas: common and divergent practices among major research universities and public sector institutions," The Journal of Technology Transfer, vol. 42, no. 6, pp. 1307-1333, 2017.

[65] L. Xing, M. Xue, and M. Hu, "Dynamic simulation and assessment of the coupling coordination degree of the economyresource-environment system: case of Wuhan city in China," Journal of Environmental Management, vol. 230, pp. 474-487, 2019.

[66] Q. Gao, F. He, A. Moosa, and Q. Lv, "The efficiency of technology transfer in Chinese key universities," Journal of Scientific and Industrial Research, vol. 78, pp. 582-588, 2019.

[67] F. Li, S. Zhang, and Y. H. Jin, "Sustainability of university technology transfer: mediating effect of inventor's technology service," Sustainability (Switzerland), vol. 10, p. 2085, 2018.

[68] W. He, Z. Lin, and R. Hao, "Analysis of regional competitiveness in the high-tech industry," Current Science, vol. 114, no. 4, pp. 854-860, 2018.

[69] B. Zhang, Y. Luo, and Y.-H. Chiu, "Efficiency evaluation of China's high-tech industry with a multi-activity network data envelopment analysis approach," Socio-Economic Planning Sciences, vol. 66, pp. 2-9, 2019.

[70] H. Chen, H. Wang, and S. Xin, "Evaluation index screening and weighting method based on information contribution rate," Scientific Research Management, vol. 41, pp. 240-247, 2020, in Chinese.

[71] F. Fan, H. Lian, and S. Wang, "Can regional collaborative innovation improve innovation efficiency? An empirical study of Chinese cities," Growth and Change, vol. 51, no. 1, pp. $440-463,2020$.

[72] M. Wang and K. X. Wang, "Exploring water landscape adaptability of urban spatial development base on coupling coordination degree model: a case of Caidian district, Wuhan," Sustainability, vol. 13, p. 1475, 2021.

[73] L. M. Zhao, L. Li, Y. J. Wu, and V. Torretta, "Research on the coupling coordination of a sea-land system based on an integrated approach and new evaluation index system: a case study in Hainan province, China," Sustainability, vol. 9, no. 5, p. 859, 2017.

[74] C. O. Fisch, J. H. Block, and P. G. Sandner, "Chinese university patents: quantity, quality, and the role of subsidy 
programs," The Journal of Technology Transfer, vol. 41, no. 1, pp. 60-84, 2016.

[75] C. M. Gross, “The growth of China's technology transfer industry over the next decade: implications for global markets," The Journal of Technology Transfer, vol. 38, no. 5, pp. 716-747, 2013. 